\title{
Exploring the Cloud Computing Loop in the Strategic Alignment Model
}

\author{
Belitski, Maksim ${ }^{1}$; Fernandez, Valerie ${ }^{2}$; Khalil, Sabine ${ }^{3}$, Li, Weizi ${ }^{1}$ and Liu, Kecheng \\ ${ }^{1}$ Henley Business School, University of Reading, Whiteknights campus, Reading, RG6 6UD, \\ UK \\ ${ }^{2}$ Department of Economic and Social Sciences, Telecom ParisTech, 46 Rue Barrault, 75013 \\ Paris, France \\ ${ }^{3}$ ICD - International Business School, 12 rue Alexandre Parodi, 75010, Paris, France \\ m.belitski@reading.ac.uk, valerie.fernandez@telecom- \\ paristech.fr, skhalilegroupe-igs.fr, weizi.li@henley.ac.uk, \\ k.liu@henley.ac.uk
}

\begin{abstract}
Since its emergence, Cloud Computing (CC) has revolutionized organization through offering them a large range of easily accessible, scalable, and non-expensive services. As CC has been gaining popularity, it has an impact on the strategic and operational level of every organization. Thus, wondering about its impact on the organizational strategic alignment is a must. This study develops the Cloud Computing framework of Strategic Alignment Model, where strategic fit between operational and strategic levels in organization is achieved through cloud-enabled multiple iterative processes. The study aims to understand whether cloud computing increases operational and strategic efficiencies of organization and if yes then how. After presenting the theoretical background, the hypotheses, and the built cloud framework, we discuss the way strategic alignment theory helps us to better understand how information flows within the strategic fit of business and IT and what is the role of CC in it. This study addresses the strategic alignment as well as the cloud computing literature.
\end{abstract}

Keywords: Cloud Computing, Strategic Alignment, Operational Management, Validation

\section{$1 \quad$ Introduction}

Although an intense research has been done about the strategic value of information technology adoption using resource-centered view [1] the contingency-based view [24], semiotic framework [5] and leadership and management view [6-8], there is a paucity of research assessing a value created by cloud computing technology (CC) [9] and the mechanism of $\mathrm{CC}$ adoption in organization [5]. Cloud computing, referring to information technologies enabling convenient, on-demand network access to a shared pool of configurable computing resources and has fundamentally changed the way companies operate and co-create value [10-13]. As a transformative technology, CC 
has changed various aspects of business and social interactions in the way businesses operate, exchange data and engage with customers [9]. To better understand a value creation process enabled by $\mathrm{CC}$, more theoretical underpinning and practical evidence is required regarding the role that $\mathrm{CC}$ plays in improvement of the organization's operations, innovation, efficiencies as well as used to design a robust IT and business strategy. It is important to operationalize previous findings and results that are focused on issues associated with information technology adoption and a framework to analyze it $[4,14]$.

$\mathrm{CC}$ continues revolutionizing the ways business collect, process, analyze, review and manage information [15-16] with a special attention on consistent measurements [17] of returns associated with CC by organization [18]. We use the term organization in this study to further emphasize the vast and far-reaching impact of cloud technology investments which goes beyond a separate business unit such as organization or business.

Although interest of IS scholars and practitioners on the cloud's impact on organizational operations and performance has been growing, the prior research related to $\mathrm{CC}$ and efficiencies has primarily focused on adoption or operations, cost reduction, exploiting the IT resource mobility offered by the $\mathrm{CC}$ as well as other supplementary technologies. There has been little attention paid to $\mathrm{CC}$ as an asset but also a strategic tool, creating more agile and flexible IT infrastructure, business operations, skills, administrative infrastructure and IT architecture. CC is likely to result in changes in administering and operations; higher rate of new product development, higher engagement of executives in IT investment and management decision-making, business growth, meticulous design of IT and business strategy within budget, time and scope. These and other important issues related to $\mathrm{CC}$ have not been explicitly discussed within the Strategic Alignment (SA) framework [14, 19, 20] with a lack of evidence on how information flows to achieve strategic fit and integration [21]. Neither has been investigated the interplay between $\mathrm{CC}$, strategic operations and strategic performance of organization with the published work being fragmented and incomplete [20].

CC can play a major role in SA in organization described in [14] as the difficulties of achieving alignment for professional organizations; the limitations, organizations have in being agile; the rationale for acquiring technology and determining IT skills; the imperative meaning that CIOs attribute to IS alignment. To demonstrate the role that CC plays ion IS in organization we build on Information Systems (IS) [4, 22], Strategic Alignment [23-27] and organizational performance literature [28, 29].

This study makes the following contribution. We develop and test the "cloud-enabled mechanism of validated learning" embedded into IS alignment model. More specifically we develop the Cloud Computing framework of SA, where strategic fit between operational and strategic levels in organization is achieved through cloud-enabled multiple iterative process.

This study offers practical implications for managers to better understand the complementary nature of new technologies and CC's embeddedness in Strategic Alignment Model. Understanding how information is collected, assessed, distributed and analyzed should enable decision-makers to design more effective and robust business and IT strategy. We argue for the need to study the continuous organizational adaptation of evolving $\mathrm{CC}$ because of the challenges such technologies pose for users, as well as the operational capabilities and strategic skills they demand. 


\section{Theoretical Background and Hypotheses}

\subsection{Strategic Alignment Lens in Cloud Computing Framework}

Cloud computing is as a strategic asset and tool rather than a service [9]. In order to assess the impact of CC, managers need to be aware that it has become another strategic asset embedded in strategic alignment of IT and business [5, 9, 19, 20, 23]. Being attributed to an improvement in data sharing, technology standardization and infrastructure, administering and operations, $\mathrm{CC}$ is further associated with the ability in delivering new projects and applications within budget, time and scope, changing strategy and engaging different stakeholders [9]. It changed the paradigm of IT investment moving from operational expenditure (OPEX) to capital expenditure (CAPEX) and decreased usage of hardware [12, 16, 31, 32, 33].

Furthermore, CC is related to greater scalability, flexibility and operation ability. This enables organizations to improve organizational infrastructure and business services $[31,32]$. The ubiquitous nature of $\mathrm{CC}[31,32,34,35]$ enables greater alignment of business and IT strategies, where different business units can integrate and use cloud solutions anywhere and anytime. On the one hand, $\mathrm{CC}$ is thought to be a reliable source when organizations acquire standardized solutions from trusted providers, therefore once adopted by organisational and IT infrastructure it affects strategic choices made by executives and related to investment in IT and business [31, 34].

To build our theoretical framework on the role of cloud technology adoption in organization we use Strategic Alignment lens [30, 36]. First, we conducted a detailed literature review to identify relevant theories of alignment and validated learning. Our extensive literature search failed to unearth theories addressing strategic alignment between strategy and technology with the important stage of validated learning. This is important when investigating the fit and integration between strategy and operations $[21,23,30]$.

\subsection{Stages of Theoretical Framework}

Our theoretical framework consists of three stages associated with Apprehension of Information, Unitization of Information, and Validated Learning. All three stages rely on 'continuous adaptation and change' within SA model [23]. Our theoretical framework illustrates various steps in information gathering, conceptualization, optimization, implementation and validated learning.

At the first stage, apprehension of Information is done through generating insights, gathering information and a process of conceptualization. It is associated with people gaining knowledge and experience through using CC for operations and as a part of IT infrastructure development. Employees who access CC may not immediately understand CC solutions until they have experienced them. Apprehension of information 
stage is about 'finding the answer' where 'finding' is something more than mere retrieval of information. It is about adoption and use of CC in daily processes. This changes the cognition of users, focused on pure knowledge acquisition by experiencing and absorbing it $[28,37]$. In addition, it requires generating and conceptualizing. Generating involves getting $\mathrm{CC}$ in place for operational and IT infrastructure. Generative thinking involves imagining possibilities where $\mathrm{CC}$ could be applied to automate processes and increase efficiencies. It further requires, questioning, sensing new opportunities and viewing IT and business processes from different perspectives and gathering information through experience and validated learning from previous iterations. The process of decision-making at this stage starts with exploring options (divergence), selecting and continues with exploiting and applying solutions with conceptualizing (convergence). The ambiguity of $\mathrm{CC}$ on alignment-operational should be pinned down. Conceptualizing results in putting new ideas together on how to use CC more efficiently.

At the second stage, Utilization of Information features the outcomes of cloud adoption and performance. At this stage, IT and business strategy can be designed reflecting on information received at stage one through optimizing and implementing strategy. It demands decision makers to apply knowledge obtained at the first stage to design strategy. They may either design a brand-new strategy, or update the existing strategy in light of changes in business processes and IT infrastructure triggered by CC. At this stage optimisation and implementation are required. Optimisation gains understanding of CC for organizational and IT strategy. This results in developing practical solutions and plans from abstract ideas, trends and insights. Given a well-defined solution, decision makers should be able to sort through large amounts of information to pinpoint the critical factors and processes where cloud is required. They should be confident in their ability to make a sound, logical evaluation of transformative impact of CC and integrate those processes. Finally, implementation is execution of designed strategy. Implementation of strategy requires complete understanding of how business operations and IT infrastructure are going to be affected by the CC. In case of strategy complete or partial failure or as a result of changes in external environment (e.g. technology, institutions, market competition, etc.), decision makers need to be agile and respond quickly. This requires transition from the second to the third stage of "Validated Learning".

At the third stage, Validated Learning measures the strategic efficiencies on both sides of IT and business. At this stage the performance is assessed and the application of $\mathrm{CC}$ is redesigned adjusting to environmental changes or addressing gaps in performance outcomes. Validated learning offers two choices: first, either retaining IT and business strategy or revising the way $\mathrm{CC}$ is implemented. At this stage the use of CC will be reshaped and redesigned to feedback to management in order to improve the efficiency of the next round of iteration of the cloud-enabled loop.

\section{The Model}

When an organization learns through development, implementation, measurement and feedback, the impact of CC could become more pronounced and ubiquitous [9]. This permits us to measure its value more distinctively. In particular, we can measure the 
transformative impact of CC by analysing its relation to innovation, strategy, development of new products and services, standardizing and sharing data, management operations, changes in processes.

Fig. 1 illustrates the place of CC within SA model which can be considered in terms of three interdependent dimensions of cloud-enabled validated learning loop. The first stage in a loop represents the Apprehension of Information through cloud adoption to improve organisational and IT infrastructure and processes. The second stage represents the Utilization of Information received from operations. Both stages are preconditioners to the third stage of Validated Learning which enables the assessment of CC efficiencies and advises action.

The theoretical framework supports the interplay between operations, strategic efficiencies and use of CC. The mechanism is iterative and can be described in stages as adopt - measure - learn - rebuild. The loop changes the way SA works, Strategic alignment is seen a process of continuous adaptation and change which could be achieved both through iterations and technology-enabled experimental learning [14, 23].

\section{Discussion and Conclusion}

Cloud computing marks a paradigm shift in the way business is done and is greatly associated with delivering high quality and timely service to customers.

The major benefits of $\mathrm{CC}$ found in this study are as follows. First, investing in $\mathrm{CC}$ enables rapidly easing the administering and operating processes within the organization and with external partners; second, it reduces cost of network maintenance, information exchange; third, CC decreases time solution and new products are developed; fourth, it improves security and compliance; and finally, it enables global deployments of solutions and faster decision making.

Building on the extent literature $[4,13,23,27]$ this study develops the Cloud Computing Framework of Strategic Alignment Theory by theoretically discussing and empirically validating the cloud-enabled mechanism embedded into business and IT alignment within SA model of organization [23]. More specifically, we established and described three distinctive stages of cloud's transformative impact on organizations: first, Apprehension of Information through cloud adoption to improve organisational and IT infrastructure and processes; second, Utilization of Information received from operations and deciding on business and IT strategy; third, Validated Learning which enables to assess the role that CC plays in operations and strategic fit and advise changes. When an organization learns through generalization, development, optimisation implementation and validated learning, the impact of $\mathrm{CC}$ could become more pronounced and ubiquitous facilitating both operational and strategic levels of organization.

Our contribution in the scholarship of IS and SA is as follows. First, we applied Strategic Alignment lens to demonstrate how each of three stages of cloud-enabled validated learning process contribute to business operations and IT infrastructure, business and IT strategy. Each of three stages forms a cloud-enabled loop of validated learning used by organizations as part of their "adopt - measure - learn - rebuild" strategy. Second, our methodological contribution is in applying a multi-level mixed method and used various sources of data to test our theoretical framework. 
The future research will need to focus on evaluating to what extent CC changes strategic integration between IT infrastructure and business operations as well as between IT and business governance. Scholars would also like to know how investment in CC will to affect strategic fit between IT and business of economic agents when their performance and operations are interconnected, such as digital ecosystems. It may appear that strategic alignment of organization is not any more dominant unit of analysis, and strategic alignment of an ecosystem could be viewed as an alternative unit of analysis. In a new digitized economy, when operations and efficiencies of ecosystem players are complementary, $\mathrm{CC}$ is likely to benefit the entire ecosystem of inter-connected organizations, rather than a focal organization. This is because $\mathrm{CC}$ makes access, processing, sharing and transforming data faster, easier and more secure. Future research needs to offer theory and implications on how CC will support operations and efficiencies of inter-connected organizations. 
Fig. 1. Theoretical Framework of Cloud enabled Validated Learning in Strategic

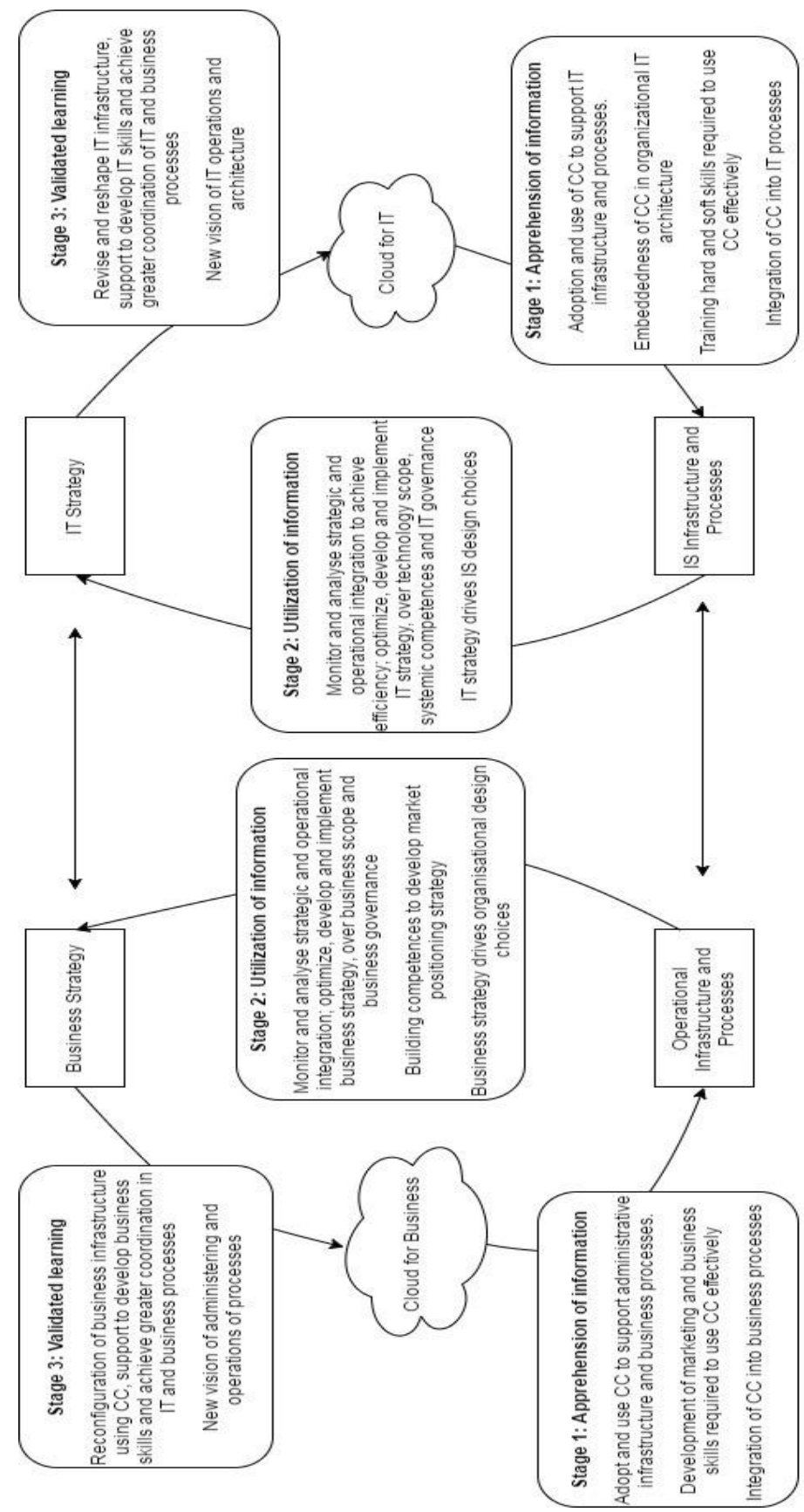




\section{References}

1. Barney, J.: Frim resources and sustained competitive advantage. Journal of Management 17, 99-120 (1991).

2. Fry, L. W., Smith, D. A.: Congruence, contingency, and theory building. Academy of Management Review 12(1), 117-132 (1987).

3. Tosi, H. L., Slocum, J. W.: Contingency theory: Some suggested directions. Journal of Management 10(1), 9-26 (1984).

4. Oh, W., Pinsonneault, A.: On the Assessment of the Strategic Value of Information Technologies: Conceptual and Analytical Approaches. MIS Quarterly 31(2), 239-265 (2007).

5. Mingers, J., Willcocks, L.: An integrative semiotic methodology for IS research. Information and Organization 27(1), 17-36 (2017).

6. Wang, P., Ramiller, N. C.: Community Learning in Information Technology Innovation. MIS Quarterly 33(4), 709-734 (2009).

7. LEAD: E-Leadership Skills for Small and Medium Sized Enterprises project. European Commission, Directorate-General for Enterprise and Industry online publication 15 July (2014).

8. Li, W., Liu, K., Belitski, M., Ghobadian, A., O’Regan, N.: e-Leadership through strategic alignment: an empirical study of small and medium sized enterprises in the digital age. Journal of Information Systems (2016).

9. Willcocks, L., Venters, W., Whitley, E.: Moving to the cloud corporation. Palgrave, London (2014).

10. Gold, J.: Protection in the cloud: risk management and insurance for cloud computing. Journal of Internet Law 15(12), 24-28 (2012).

11. Juels, A., Oprea, A.: New approaches to security and availability for cloud data. Communications of the ACM 56(2), 64-73(2013).

12. Yeboah-Boateng, E. O., Essandoh, K. A.: Factors influencing the adoption of cloud computing by small and medium enterprises in developing economies. International Journal of Emerging Science and Engineering 2(4), 13-20 (2014).

13. Winkler, T. J., Benlian, A., Piper, M., Hirsch, H.: Bayer HealthCare Delivers a Dose of Reality for Cloud Payoff Mantras in Multinationals. MIS Quarterly Executive, 13(4) (2014).

14. Silva, L., Figueroa, E., González-Reinhart, J.: Interpreting IS alignment: A multiple case study in professional organizations. Information and Organization 17(4), 232-265 (2007).

15. Noor, T. H., Sheng, Q. Z., Zeadally, S., Yu, J.: Trust management of services in cloud environments: Obstacles and solutions. ACM Computing Surveys (CSUR) 46(1), 12 (2013).

16. Garrison, G., Kim, S., Wakefield, R. L.: Success factors for deploying cloud computing. Communications of the ACM 55(9), 62-68 (2012).

17. Brynjolfsson, E.: The productivity paradox of information technology. Communications of the ACM 36(12), 66-77 (1993).

18. Barua, A., Konana, P., Whinston, A. B., Yin, F.: An empirical investigation of net-enabled business value. MIS quarterly 28(4), 585-620 (2004).

19. Preston, D. S., Karahanna, E.: Antecedents of IS strategic alignment: a nomological network. Information Systems Research 20(2), 159-179 (2009).

20. Gerow, J. E., Grover, V., Thatcher, J. B., Roth, P. L.: Looking Toward the Future of ITBusiness Strategic Alignment through the Past: A Meta-Analysis. MIS Quarterly, 38(4), 1059-1085 (2014). 
21. Coltman, T. R., Tallon, P. P., Sharma, R., Queiroz, M.: Strategic IT alignment: twenty-five years on. Journal of Information Technology, 30(2), 91-100 (2015).

22. Sabherwal, R., Chan, Y. E.: Alignment between business and IS strategies: A study of prospectors, analyzers, and defenders. Information systems research 12(1), 11-33 (2001).

23. Henderson, J. C., Venkatraman, N.: Strategic alignment: leveraging information technology for transforming organizations. IBM Systems Journal 32(1), 4-16 (1993).

24. Hirschheim, R., Sabherwal, R.: Detours in the path toward strategic information systems alignment. California Management Review 44(1), 87-108 (2001).

25. Peppard, J., Campbell, B.: The Co-evolution of Business/Information Systems Strategic Alignment: An Exploratory Study. Journal of Information Technology (2014).

26. Avison, D., Jones, J., Powell, P., Wilson, D.: Using and validating the strategic alignment model. The Journal of Strategic Information Systems 13(3), 223-246 (2004).

27. Aanestad, M., Jensen, T.: Collective mindfulness in post-implementation IS adaptation processes. Information and Organization 26(1), 13-27 (2016).

28. Dehning, B., Richardson, V. J., Zmud, R. W.: The value relevance of announcements of transformational information technology investments. MIS Quarterly, 637-656 (2003).

29. Devaraj, S., Kohli, R.: Performance impacts of information technology: Is actual usage the missing link? Management science 49(3), 273-289 (2003).

30. Henderson, J. C., Venkatraman, N.: Strategic alignment: a framework for strategic information technology management. Working Paper No. 190, Cambridge, MA: Center for Information Systems Research, MIT (1989).

31. Dutta, A., Peng, G. C. A., Choudhary, A.: Risks in enterprise cloud computing: the perspective of IT experts. Journal of Computer Information Systems 53(4), 39-48 (2013).

32. Armbrust, M., Fox, A., Griffith, R., Joseph, A.D., Katz, R., Konwinski, A., Lee, G., Patterson, D., Rabkin, A., Stoica, I., Zaharia, M.: A view of cloud computing. Communications of the ACM 53(4), 50-58 (2010).

33. Buyya, R., Yeo, C. S., Venugopal, S., Broberg, J., Brandic, I.: Cloud computing and emerging IT platforms: Vision, hype, and reality for delivering computing as the 5 th utility. Future Generation computer systems 25(6), 599-616 (2009).

34. Tiers, G., Mourmant, G., Leclercq-Vandelannoitte, A.: L'envol vers le Cloud: un phénomène de maturations multiples. Systèmes d'Information \& Management 18(4), 7-42 (2014).

35. Leavitt, N.: Is cloud computing really ready for prime time. Growth 27(5), 15-20 (2009).

36. Ciborra, C. U.: De profundis? Deconstructing the concept of strategic alignment. Scandinavian Journal of Information Systems 9(1), 67-82 (1997).

37. Harvey, O.J., Hunt, D., Schroeder, H.: Conceptual systems and personality organization. John Wiley, New York (1961). 